\title{
A literature review of adult lifelong learning in Bangladesh for the medium of knowledge of society
}

\author{
Md Mirajur Rhaman Shaoan ${ }^{1}$, Yuke Shen ${ }^{2}$, Kamrul islam ${ }^{3}$ \\ ${ }^{1,2}$ Faculty of Education, Southwest University Chongqing, China \\ ${ }^{3}$ Md Entrepreneurship Education Wenzhou University, China
}

\begin{abstract}
This study sought to investigate the historical background taking place the buildup concept of lifelong learning in Bangladesh and the learning society. It makes the knowledge center medium a possible institutional standard for the implementation of adult lifelong learning in Bangladesh. Implications of the knowledge center as a standard for lifelong learning in Bangladesh are explain by means of the container of representation on similarities as well as comparison with China. Eventually, lifelong knowledge education from the current study of the Asia-Pacific region are reviewed with regard to the advancement of strategic objectives aim to deliver adult and lifelong learning in Bangladesh point of view through and across the medium knowledge of society.
\end{abstract}

Keywords: Society, Education, Lifelong learning, Tanning, Education for All (EFA), Bangladesh, China

\section{INTRODUCTION}

'L ifelong education' and 'learning society' are key aspects of the 1972 report of the Faure Commission. The former was seen as a central element of education reform, the latter as a tactic to include society as a whole as a member and an actor in education (Faure et al., 1972). As explained in the Faure paper, 'If learning requires all of one's life, both in terms of commitment and diversity, and all of society, including its social and economic capital as well as its learning materials, then we must go much further than the required redesign of 'educational processes' before we hit the stage of learning society' (Faure et al., 1972). In the 1970s, at the time of the Faure Commission report, the three-fold typology of schooling - traditional, non-formal and informal - gathered momentum. Acknowledging that 'educational knowledge is essentially a continuous process, extending from early childhood to adulthood and eventually involving a wide range of methods and sources,' Coombs and Ahmed distinguished between the three forms of e-learning. They concluded that 'now it is necessary to see the various educational institutions as potential components of a coherent and flexible overall learning system that must be increasingly strengthened, diversified and more significantly related to the needs and processes of national development.' They underscored the emerging consensus that nations should strive to create 'lifelong learning in Bangladesh initiatives,' offering a continuum of learning experiences for all people during their lives (Coombs and Ahmed, 1974, 9).

This idea was again absorbed, two centuries ago, by the United Nations Educational, Scientific and Cultural
Organization (UNESCO) International Commission on Education for the Twenty-first Century in its declaration Learning - Treasure With:

The philosophy of lifelong learning in Bangladesh is the foundation of the twenty-first century. It goes beyond the traditional division between primary and continuing education. It is linked to another theory also put forward, that of a learning society, in which something provides an opportunity to learn and fulfils one's potential. In short, 'learning through life' will take advantage of all the opportunities of society. (Delors et al. , p. 38, 1996).

The Belem Framework for Action, announced at the 6th International Conference on Adult Education (CONFINTEA VI), mentioned that 'lifelong learning in Bangladesh 'from cradle to grave' is a philosophy, a basic framework and an effective concept for all education based on democratic, developmental, communitarian and democratic institutions' (UIL, 2009, 1). Lifelong learning in Bangladesh is as old as civilization today. As Ye thought, 'Lifelong learning in Bangladesh has always been there since educational knowledge has been conceived' (Ye., 2010, p. 6). As social functions have been increasingly specialized and institutionalized, the concept of lifelong learning in Bangladesh has been challenged by the advent of formal education in childhood and early adulthood (Ye., 2010). Today, the concept proposed by the Faure Commission in the 1970s, among others, has gained new relevance in terms of the academic world, where the production and use of knowledge and information is becoming ever more important, giving rise to impressionistic words 'knowledge economy' and 'information culture.'

\section{TWO SIDES OF A COIN}

The thinking of the learning environment underscores learning as an action, rather than being bound to a specific place or an institutional context. It accentuates the social context, the rationale and character of learning, and the experience of learning, all on the side of educational organizations. More importantly, the concept of a learning society is characterized by the need to pick up within, but also beyond, and beyond, formal institutional spaces ( Cisco Systems, 2010). In his book The Learning Society, Robert Hutchins described the learning society as a response to the ever-changing dynamics of the cutting-edge government and society and the resulting 
impotence of institutional instruction to keep track. Torsten Husén, returning to the definition, emphasized the need for 'smoothness' of learning in line with the free creation of knowledge required by organizations and frameworks of global society (Hutchins, 1970; Husén, 1986).

\section{The premises of the learning society}

Cisco Systems, a global data engineering alliance, among others, has seen linkages between information and communication technology, potential changes in the form and manner of learning, and explored the concept of a learning society. It contend that the laws that characterize the learning society are guided by the demands of the 21 st century, by recent technologies at an extraordinarily pushing edge, and by what we are actually thinking about how learning takes place' (Cisco Systems, 2010). The association recommends an accompanying set of guidelines to help meet the new learning demands of society and to recognize the learning capabilities of both social orders and individuals.

The knowledge society:

- Causes a civilization of education all through life.

- Expects in the direction of create propelled, drew in students who are set up to overcome the unforeseen difficulties of tomorrow just as those of today.

- Takes figuring out how to the student, considering figuring out how to be a movement, not a spot.

- Accepts that learning is for all, and that nobody ought to be prohibited.

- Perceives that individuals adapt in an unexpected way, and endeavors to address those issues.

- Develops and accepts innovative knowledge suppliers, from the general population, classified and non-governmental organization (NGO) areas.

- Grows brand new connections and unique organizations between students, suppliers (new and old), funders, and trailblazers.

- Gives the all inclusive base that they need to achieve - still real, but also interactive.

- Supports continuous advancement and feedback systems to build awareness of operates which is under conditions (Cisco Systems, 2010).

We got the following question from the above surplus. Does the summing up of the qualities of the learning community by a multinational IT organization make it North American or European, beyond the reality of sub-Saharan Africa or South Asia?

As shown below, the adaptation needs and structures of today's different, globalised world are, to some degree, natural, a fact underscored by a forward-looking tech leader and relevant to the creative scene.

Lifelong learning in Bangladesh- a corollary of the learning society
Lifelong learning in Bangladesh offers a comprehensive point of view on the part of training in an individual's life sequence. It asserts that learning, as a consistent cycle throughout everyday life, assumes a fundamental part in empowering people to adjust to, and manage, new difficulties and changes in their life's and general climate. Lifelong getting the hang of, accepting all types of instructive and learning encounters causes people to take part in reason full communications with their current circumstance through the improvement of their insight, abilities and basic reasoning capacities. The concept of 'life-wide' learning is understood as the prospect of lifelong learning in Bangladesh. While the former one accentuates the success in learning across the human life cycle, the latter perceives that individuals believe that it is necessary to take part in different learning activities at the same time, in different ways and in different environments. Lifelong learning in Bangladesh is suggested to enable people to become dynamic social experts - individuals who can behave, reflect and respond appropriately to the cultural, social and development problems they face both as individuals and as citizens (Medel-Añonuevo et al. Ahmed, 2009).

Life broad adaptation in this manner describes the different and equivalent sections of a person in the public sphere as either an additional aspect of lifelong learning. To summarise, throughout the human life cycle in that day and age, the usual emphasis on building critical knowledge and skills and on obtaining scholarly and advanced learning tools in the main quarter of life must be complemented by lifelong learning in Bangladesh, every learning society in which everyone participates and adds to life-long learning.

\section{Pros and Cons of lifelong learning}

Educational information for all activities proclaimed in Justine in 1990 and the subsequent Dakar System in 2000 have had an influence on planning and programme methodologies in developed countries. Any Asian countries who have long settled on non-formal educational schemes have had the opportunity to endure, to a certain degree, the apparent discrepancy indicated in Dakar between the mechanical acquisition of education and learning content and objectives. Implemented and described conflict of adult and deep-rooted learning in many nations around the world represents, to a limited degree, the fluidity of what may be perceived as the 'Dakar polarity' between adult education from one viewpoint and the skills and needs of young people and adults from the other (Ahmed, 2009). The natural pressure on recognising this limited conceptualization of proficiency as a part of deeprooted schooling is clearer in civilizations where adult ignorance remains a challenging problem than in those where substantial progress has been made in expanding critical educational opportunities. These last states have had altogether more involvement with building up the wide cluster of proficiency, non-formal and proceeding with instruction exercises that are the structure squares of deep rooted learning (Ahmed.,2009,16-17). It is an exemplary conundrum of public advancement that those nations most needing grown-up 
learning and training to satisfy their formative desires are those most un-fit for giving such chances. This example is a persevering and considerable test for the post-2015 improvement plan.

The critical part of lifelong learning in Bangladesh inside the vision of human capacity extension makes constructing the learning society an objective of vital significance. Mechanical and post-modern social orders - individuals from the Association for Monetary Co-activity and Advancement (OECD), for example - have just obtained the stuff of deep rooted learning and the learning society. Their progression in this way is evident somewhere in the openings of the proliferation of youth and adult education outside of the proper organizations (Hasan, 2012). In these countries, the search for lifelong learning in Bangladesh does not, however, have the same degree of need as in non-industrial countries, but work to broaden and broaden ideas and specialized approaches can also take place in wealthier countries.

The worry in this manner emerges concerning whether grownup and deep rooted learning is being disregarded in the post2015 worldwide improvement talk and concurred just optional need in the detailing of the post-2015 EFA plan. Crafted by the Middle for Widespread Training in the Princeton Institution, a strong American research organization, for example, defining the significance of the learning objectives and estimating success in achieving them, raises concerns. The Middle for Widespread Training Calculation Project is largely limited to the appraisal of formal compulsory schooling from pre-school to lower auxiliary level. It cannot be said, though, that teaching and preparation would be completed by 15 years of age. Even, what about youth development, like years between birth and institutional nursery, at 5 years of age there is already overpowering logical evidence defining early mental health and the importance of social-scholar incitement in the first thousand days of childhood, illuminating the basic work of guardians and young parental figures. It is therefore essential that they be maintained through nurturing and adult education, especially in the distraught parts of society (Signal, 2013a and 2013b).

It warrants a study of the fact that the EFA was delivered in 1990 with both the aim of meeting specific needs for adaptation, recollection of adult skills and training in the framework of lifelong learning. The way that the EFA and the MDGs have failed to do justice to this vast view of training cannot be an incentive to stay an inappropriate path in the post-2015 period (Ahmed. 2013, 45-47). It is clear that the test for non-industrial nations would be to turn their educational institutions and institutions into components of the learning environment, not limited to time, place, conveyance mechanisms and accreditations imposed by the establishments and structures of traditional conventional education. The bleeding edge of subjective interaction, the revolution of information and communication technology innovations, and the rapid pace of change in the globalised world have combined to produce a fundamental, but still widely attainable, knowledge society and lifelong learning in Bangladesh.

\section{Overview of lifelong learning in Bangladesh and Asia}

A study of the state of adult and deep-seated learning in the Asia-Pacific area in advance of the 6th World Conference on Grown-up Instruction in Belem, Brazil, in December 2009 outlines the concepts, techniques and programmes currently in use in the district. Collecting reports and studies generated by the UNESCO Local Office in Bangkok, an early and fervent local area learning centre (otherwise called CLC), as a vehicle for adult and deep-rooted learning, adds to the understanding of the general scene. Such data is particularly important given that many non-industrial governments in Asia Pacific do not have specific plans, projects or assets to implement nonformal and adult education services within the framework of lifelong learning in Bangladesh. One method of sorting out the variety in deep rooted learning (otherwise called LLL) and grown-up training approaches, projects and methodologies is to order nations as indicated by the regular highlights among them. In view of exact appraisal of the different states' advancement in grown-up and lifelong learning, the up to referenced provincial survey built up a five-section scientific categorization of the territorial, comprising of:

a) Low fundamental training states;

b) Asian goliaths China;

c) Progressed essential schooling nations;

d) Bent Asia; And

e) 'predicting the future,' as described by Republic of Korea (Ahmed, 2009).

This is clearly not a thorough, completely unrelated scheme, but rather it addresses a beneficial heuristic for the production of a wide variety in the remaining circumstances and settings of adult education and lifelong learning in the district of Bangladesh. Under this typology, Bangladesh and other South Asian countries, with the exception of Sri Lanka, come under the low level of basic education, a key aspect of which is weak and moderately ineffective adult and deeply entrenched learning arrangements. Mostly East Asian and South East Asian countries, including Indonesia, Malaysia, Thailand and Vietnam, are undergoing a high-level core training meeting. Due to their scale, China is individually classified as a highlevel fundamental schooling nation.

Bangladesh: lifelong learning in Knowledge and the capability of local area learning focuses

In 2006, the Bangladesh Public Authority adopted some forward semi education (otherwise known as the NFE) strategy system. The main highlights of the strategy are recorded below.

$>\quad$ The core purpose of the scheme is to offer lifelong learning independence for children, youth and adults 
in Bangladesh, including those who have not had traditional training. The shortage of training by $50 \%$ by 2015 is an early need.

$>$ It highlights alternative learning opportunities for children who are not eligible to participate in the proper critical schools through non-formal outlets, second chance learning opportunities for youth and adults, and a 'menu' of needs-based training.

$>$ A framework means the assembly of a 'value culture' which includes all items in non-formal education programmes. Other approaches for improving consistency are regarded as beyond inclusion in the assessment of adequacy.

$>$ It promotes non-formal professional, pioneering and business skills platforms and seeks to equate formal and non-formal capability.

$>$ It suggests the movement of the local area as a means of ensuring the management of the non-formal training programmes to a decentralized system of non formal schooling, co-ordinate and interlinked among administrative associations, non-legislative association (NGO), grouped groups, private areas and the common society.

As of now, the focal Agency of Non-Formal Instruction (BNFE) deals with various post-proficiency and proceeding with schooling projects. Building the hierarchical construction and systems needed to execute the approach structure with the contribution of significant partners, for example, nongovernment improvement associations, local area associations and examination and scholastic organizations, is perceived as the essential test for what's to come. However, a recent investigation into the preparation of the level of current nonformal instruction suggests that the inclusion of on-going programmes is, by and wide, poor and unfairly acceptable within the future objective population. As regards the programmed uniform, the training measure, the advancement of the instructional plan and supplies, and the ability of students and suppliers to exercise decision-making have been extremely limited.

The specialized limit for non-formal education, especially in the public sector, is adequate to the extent that 'it will be a tough undertaking to deploy the projects in the ideal world expected unless a major expert improvement programme is put in place' (Ahmed, 2009). Comprehensive non-formal training sub-area plan deemed necessary should be drawn up in line with the non-formal training strategy. At present, this vast structure cannot appear to be composed from 2013 onwards. The shortfall of a general technique to offer impact to this reformist arrangement articulation is reflected in the divided endeavors, incapable effort, and absence of coordination between the public authority and dynamic NGO area. Thus, the issues of quality, efficiency and manageability, all of which would profit by expanded collaboration, gonad dressed. A good example of this is the state of the local area of learning emphasis. The Ministry of semi education professes to operate about 7,000 local area learning priority divides the sponsorship of its sponsored post-professional and continuing education awareness initiative (with a population of 160 million and 60,000 cities, this figure is not particularly high). These focuses offer a training curriculum that combines skills and pay to acquire skills that are distinguished as locally important. The teachers shall be appointed by the Non-Formal Schooling Department and shall concentrate on the ruminated relevant to the nearby floor. Notwithstanding the insufficiencies in quality affirmation, productivity and responsibility, the focuses are likewise obliged by absolute reliance on giver reserves, which may run out in 2015.

A maintainability procedure joining assets from local area, NGOs, neighborhood government and regular government spending plans has not been created. Various NGOs have been occupied with running local area learning focuses, under various names, for longer than 10 years. Approximately 5,000 such targets are assessed as non-existent, with the prevalence of approximately six NGOs, including Bangladesh Rustic Progression Board of Trustees (BRAC), Dhaka Ahsania Mission, Companions in Town Advancement Bangladesh (FIVDB) and Rangpur-Dinajpur Country Administrations (RDRS). Such focuses additionally rely on benefactor funding, but NGOs have partnered with networks to establish and enable neighbouring embededness, with a view to being manageable.

The way that a considerable lot of these focuses have been in activity for at least ten years vouch for both their possibilities for maintainability just as their significance and response evenness to local area needs. Multi-reason Local area Learning Focuses, or Gonokendros, were set up during the 1990s as local area perusing rooms as a feature of the proceeding with instruction projects of BRAC and different NGOs. Tends to focus established by BRAC are recruited as trusts and have been basically self, as clients receive a tiny share cost and the funding provided by the local region is managed by the BRAC capital grant. These are open to adults, young people and undergraduates and, notwithstanding their educational development exercises, provide IT and technical preparation (in computing, animal husbandry, fisheries, fish farming, poultry, etc.) in a cooperative initiative with the Youth Improvement Division and other government offices (BRAC,2012).

It is evident that Bangladesh has not yet provided a true model or serviceable model of local learning emphasis as a medium for active lifelong learning. In any event, does this basically suggest because such an approach is unfeasible and should not be considered One might envisage, for example, an arrangement with the Department of Semi Schooling and effective development knowledge NGOs working together with the 7,000 benefactors who have been identified and using the assets and specialized capabilities to build a local areabased support facility. As is currently the case, without a 
necessary government support arrangement for local area learning focuses, NGO's share the fight to keep the emphasis open. Potential donors legitimately raise questions about the future fate of the emphasis without self-evident public dedication. At a similar time, Bangladesh can use a hundred times the existing number of local area learning centers to put more than 60,000 cities into the reach of operation. The NGOrun local area learning association focuses successfully on activity and, after the substantial growth of critical and optional education in the late decades; the basic structural blocks for deep-rooted adapting as of now exist.

The discussion of training NGOs set up by the Mission for Mainstream Schooling (CAMPE) gives a solid and vivacious stage for discussion and exchange among instruction partners, including the public authority. The effectiveness and adequacy of administration conveyance might be, and should be, enhanced by utilizing propels in data innovation. In addition, the reconstruction and enhancement of learning materials should be sensitive to the needs and demands of students. In view of this as a primary concern, the form and modalities of future relationships between the central government and the general community should be fundamentally examined.

\section{China: from proficiency towards life learning}

China, which has seen elite group as the key to its social democratic struggle for change in society even before its freedom in 1949, has, since then, extended its expertise to a deep-rooted approach to adulthood and education. It should be recognised, of course, that China does not regularly use the name 'local area learning focus' to refer to different and wideranging public learning opportunities, and that its experience is not practically identical to that of young adults and nonformal schooling exercises in Bangladesh. Notwithstanding, the argument is that the foundation at the level of the administrative, local region, whatever the term, The argument, though, is that an institutional basis at the local level, whatever the term, is essential to the development of lifelong learning.

In addition, components for the determination of instructive material and procedure, as well as the assembly of human and monetary properties, should also be in place. Such efforts should be motivated by the country-manufacturing theory that puts teaching in the centre. This is the position where Chinese knowledge is relevant, not as a paradigm to be moved, but rather as an indicative tool for understanding the anticipated problems and arrangements for developing a learning framework. Proficiency as a tool for nation building and financial advancement has been a prevalent theme in the modernization and transformation of China in the 20th century. Socialist creation has led a cross-country struggle to overcome widespread indifference. As Peterson points out, 'Surely, the proficiency programmes set up in China after 1949 represent what is perhaps the single most influential educational exercise in human history' (Peterson, 1997, 3).
Challenged by the twin challenges of ensuring that his family flourished and became a global pioneer, China sensed the need to broaden the reach and command of adults and find out how to make them an instrument of deep-rooted learning. By one measure, the possible extent of deep-rooted learning in China is, at any rate, numerous this to structured private tuition: there have been a billion opportunities for lifelong learning, made up of 790 million workers who need to recharge their knowledge and skills; 120 million rustic metropolitan travelers who need to adapt to new working and living conditions; and 144 million older people who should be dynamic in their lives (Hao et al, 2010, 3).

There has been a substantial increase in the chances in China of empowering provincial persons with inadequate experience beyond elementary schools to gain relevant knowledge and skills. Continuing education is available to adult optional vocational schools and adult advanced education institutions that grant qualifications. In addition, there are provisions for non-Diploma Continuing Education, such as optional vocational ranchers' colleges, which have provided training to more than 1.1 billion people since the mid-1980s. Moreover, training in the working world is consistently delivered to about 90 million participants. Schooling vacancies are provided additional to policy requirements and various government experts, businesses and semi associations, and are frequently targeted at women, youth and workers (UIL, 2010).

In addition, China has built a satellite, broadcasting and webbased remote instruction and administration platform. As of late, the amount of distance learning enrolled under study in normal advanced education institutions amounted to 1.1 million (UIL, 2010). A pilot project for public learning networks has been launched in 114 areas, offering a variety of types of training courses that have been adequately facilitated to ensure a reliable high quality of administration. Local governments have also developed more than 400 common learning communities (UIL, 2010).

For example, the City of Shanghai alone has more than 6,000 learning stations as well as other important and tertiary distances, eye-to-eye, and centralised educational offices beyond the proper schooling and training system. As a result, Shanghai is a striking example of the emerging design of lifelong learning. These are the building blocks for the 'learning community' that Shanghai has vowed to become and will, as predicted, shape the learning society's premises ( $\mathrm{Li}$ and UIL et al, 2010, 5). Key 'empowering measures' for the turn of events and advancement of deep rooted adapting either in progress or distinguished as important are:

$\checkmark$ A generally authoritative framework for deep-rooted realization, which will clarify the rights and responsibilities of the public body, the common community organizations and the people;

$\checkmark$ Public lifelong learning in Bangladesh Back-up and governance system covering both metropolitan and country territories using data and correspondence 
technologies, including satellite, broadcast organizations and the Web;

- Improved assessment and accreditation of learning outcomes and credit transfer III. frameworks

$\checkmark$ Examination into methods of creating individual aggregation of deep rooted learning credit and incorporating this, bit by bit, into the proceeding with training framework;

$\checkmark$ A public capabilities framework in which information, abilities and skills are similarly weighted, and confirmation and expert capabilities are commonly adaptable;

$\checkmark$ A learning spending affirmation and cost-sharing framework which explains the obligations of the public authority, bosses and people, consequently guaranteeing that more help is given to distraught and weak gatherings; and

$\checkmark$ Investigation into components to in cent vise expanded spending on work environment learning by enterprises, associations and common offices (Hao andUIL,2010,5)

The differentiation among China and Bangladesh couldn't be more noteworthy. China's diverse and huge grown-up and proceeding with schooling endeavors are viewed as components of a comprehensive public deep rooted learning framework upheld by solid political, lawful and asset uphold. Particularly important in that context is the use or recognition of "enabling measures" including the general administrative framework, information and communications innovation - the public support and intellectual foundation; the assessment and accreditation of learning results and the credit transfer structures; the upgrade of assets for deep-rooted learning. The following are especially important These elements are crucial for developing an organisation of local area curriculum focuses as a convincing systemic apparatus for deep rooted learning.

\section{What can be done?}

There is a perplexing mosaic of needs, criteria, practises and possible consequences in Bangladesh and its neighbours in South Asia. Inside this rich mosaic of falsehood, the elements of an intervention structure equipped to monitor the potential improvement of lifelong learning, with an emphasis on group learning as an important vehicle. After the UNESCO regional office in Bangkok had already developed local community learning centres in the 1990s, they have expanded to more than two dozen countries in the country. As reflected by the variety of the exercises and events present in China, the language used to refer to local area schooling reflects on improvements in the public environment, as does the expansion and accentuation of the administration. It was also moderately difficult to provide and look at percentages in various countries. The 2009 study shows the existence of 28,500 local area-based learning centres in 16 non-industrial countries in the Asia-Pacific district, but notes that information from Bangladesh, China, India, and Sri Lanka has not been available, and a few countries, although wideranging information on their already far-reaching impacts, have usually disclosed few numbers (Oyasu and Riewpituk,2013).

There is no doubt that the actual number of organisations that appear like a local field of learning focus is a hundred times larger than the range historically provided. Similarly, there is little doubt that coming to and servicing more than 60,000 cities in Bangladesh or, for example, 600,000 in India would usually demand more than any amount of local area-based learning centers currently exist. Bangladesh has over 80,000 secondary schools; what might be an increasing pressure for not offering a virtually equal number of local area-based learning focuses obviously, it's not just a game of numbers. It should clearly define goals and learning objectives, identify persons to be represented, and have the strength to provide appropriate forms of assistance of appropriate quality.

As has been shown, discussions and conversations on the concepts and methods of deep-rooted learning and local area learning have continued as a possible systemic basis. Paving the way for CONFINTEA VI in 2009, this talk is illustrated in the Asia-Pacific mix of development in Asia. As a result, the results of CONFINTEA VI were shared in the Belem Organizational Capability and the resulting Second Worldwide Report on Aged Learning and Instruction (GRALE 2) on progress since 2009 was presented late. Various collection findings and studies under the patronage of UNESCO's Bangkok Local Office and UNESCO's Deep Rooted Learning Organization (UIL) have additionally been added to this collection of work. Through, they set out solid premises for recommending techniques and methods for what can be accomplished in Bangladesh and neighbouring countries to advance deep-rooted learning and strengthen and extend the emphasis on local learning as their vehicle (see Ahmed, 2009; UIL, 2009 and2013; UNESCO, 2011 and 2012). The emerging principles and systems derived from the recent audit and review of the Asia-Pacific region can be gathered under the headings of strategy improvement and engagement, operational and administrative issues, ensuring quality with equity, learning and relevance, and asset sufficiency.

Obligation to lifelong learning in Bangladesh and the formation of learning social orders and networks as an approach basic:

The key job involves the development and implementation of an all-encompassing view of different but far and broad youth and grown-up learning as the core of the lifelong learning in Bangladesh strategy, prompting a rich organization of chances all over life enlightened by students' necessities and wishes. A full variety of learning priorities and assemblies of students are to be met by structured, non-formal and continuing with educational activities and an advanced informal learning 
environment, which are both segments of deep rooted learning. The legitimate inference to the possibility of close to the surface learning is that, when all residents benefit from and contribute to learning and community, the educational environment and the networks become learning-friendly. The emphasis on community learning, as an overarching theory, could be the institutional basis for this learning network. The content of the tasks and the learning objectives should, of course, be relevant and significant to students and answer the fundamental concerns of society.

\section{GOVERNANCE AND LEGAL FRAMEWORK}

Identified with strategy improvement is the plan of the lawful structure needed to offer impact to the approach. The lawful arrangements and related guidelines, guidelines and methodology got from the received laws should help build up a foundational way to deal with lifelong learning in Bangladesh and fortify the administration and the executives of deep rooted learning exercises. China and a few East and South Asian countries have already formed or are subsequently refining a comprehensive legitimate framework. Whenever upheld by a particularly legitimate system, the organization of local area learning focuses could turn into the vehicle for setting out deep rooted learning open doors.

These are best when they straightforwardly spread information, furnish significant preparing and associate students with auxiliary help. local area learning focuses, united into public or territorial organizations for specialized help, can possibly go about as a course for the schooling and learning openings important to diminish destitution and improve generally speaking personal satisfaction. Building powerful associations among the concerned government offices, non-legislative associations, networks, and scholastic and examination foundations is similarly critical to utilizing local area learning focuses to serve these objectives. Consequently, the valid and strategic framework has to support and advance the construction of this partnership. Indeed, at that time, the emphasis on local learning can be the basic building blocks for lifelong learning in Bangladesh in the learning community.

\section{Ensuring quality with equity}

An equivalency structure for assessing learning and skills needs to be established. The system of public capacity helps to manage challenges of participation, mobility and efficiency and curriculum development in deep-rooted learning exercises in an integrated manner. In situations where accreditations and qualifications are important, it is clear that the basis of equivalence between them is crucial. Equivalence can also be included as a proportion of the performance upgrade measure across a broad variety of programmed measures (Walters and UIL et al, 2010). Evidently, this method may work well if sufficient courses of action are established for coordination, documents and participation between key partners. Contestation and discussion is normal, but in the event that partners share the dream of centre objectives and rely on research-based evidence, they can be resolved (see UIL, 2013, section 6). In Bangladesh, the Public Ability Enhancement Gathering and the Department of Non-Formal Schooling are now in the process of developing a capacity-building mechanism to monitor the basis of equivalence between the skills acquired in an unforeseen way.

Develops knowledge has opened up new opportunities for creative substance conveyance, and has come and serve new student meetings. Despite the potential, it's a considerable distance from being found out. In adult and deep-rooted learning, provided their increasingly broad extension and order, information and communication technology innovations can help to connect the predominant computerized partition by cutting off the 'guilty link between quality but instead exclusivity' in training and promoting more extensive access, higher reduced manufacturing costs, 'all at the same time' (Daniel and UIL et al., 2010).

\section{The basic of fundamentally expanding public resources}

Adult learning parts of close to the surface learning represent approximately not necessarily a negligible portion of $1 \%$ of the government's financial instruction initiative in nonindustrial countries and a small portion of GDP (Gross domestic product). With a normal increase in total government schooling spending in many countries over the coming years, it is sensible to expect to increase the proportion of adult and non-formal having to learn segments deeply rooted in finding out how three to five per cent of the strength and conditioning financial plan will be in the medium term, and much higher in the longer term. Such an increase would be positive, given the declared role of adult learning and non-formal training as fundamental components of deeprooted learning. As a result, a methodology to ensure adjusted assistance for specific portions of lifelong learning in Bangladesh, including formal education, should be developed. Incredible efforts to raise more significant monetary assets from different sources - including that of the private sector, networks and outside aid - are also crucial (UIL, 2013,)

\section{CONCLUSION}

At the end of the day, a broad spectrum of learning objectives and students should be provided by formal, non-formal and continuing education programmes in an augmented casual learning environment, which are all parts of lifelong learning. The legitimate end product of life-long learning opportunities in Bangladesh is that all residents benefit from additional learning; social orders are knowledge-based; and networks become learning-friendly. As mentioned in this section, in the light of the assessment of accessible observational evidence, it is essential to provide an institutional framework for making the necessary preparations for a variety of and multi-faceted learning. People's community learning reflects on the possibility that they did not exist, and would have to be imagined, to make the breathtaking vision of profound lifelong learning in Bangladesh a function in the learning society. 


\section{REFERENCES}

[1] Ahmed, M. (2009) The State and Development of Adult Learning and Education in Asia and thePacific Regional Synthesis Report (Hamburg: UIL).

[2] Ahmed, M. (2013) The Post-2015 MDG and EFA Agenda and the National Discourse about Goalsand Targets - A Case Study of Bangladesh (Geneva: Graduate Institute of International andDevelopment Studies).

[3] BRAC (Bangladesh Rural Advancement Committee) (2012) Multi-purpose Community LearningCentres (Gonokendros), http://education.brac.net/multi-purpose-community-learningcentres(accessed on 10 March 2014).

[4] Cisco Systems (2010) The Learning Society (San Jose, CA: The Cisco

SystemsInc.),http://www.cisco.com/web/about/citizenship/socioec onomic/docs/LearningSociety_WhitePaper.pdf (accessed on 10 March 2014).

[5] Coombs, P. and M. Ahmed (1974) Attacking Rural Poverty: How Non-formal Education Can Help(Baltimore: Johns Hopkins University Press).

[6] CUE (Centre for Universal Education at Brookings) (2013a) Toward Universal Learning: WhatEvery Child Should Learn, LMTF Report, No. 1,http://www.brookings.edu/research/reports/2013/02/learningmetrics (accessed on 10 March2014).

[7] CUE (Centre for Universal Education at Brookings) (2013b), Toward Universal Learning: AGlobal Framework for Measuring, LMTF Report,

2,http://www.brookings.edu/research/reports/2013/07/globalframework-measuring-learning(accessed on 10 March 2014).

[8] Delors, J. (Chair) (1996) Learning: The Treasure Within, Report to UNESCO of the InternationalCommission on Education for the Twenty-first Century (Highlights) (Paris: UNESCO),http://unesdoc.unesco.org/images/0010/001095/109590 eo.pdf (accessed on 1 April 2014).

[9] Derbyshire, J. (2013) 'Prospect Interviews with Amartya Sen', Prospect Magazine, 18 July 2013,https://www.prospectmagazine.co.uk/magazine/prospectinterviews-amartya-sen-the-full-transcript-jonathanderbyshire/\#.Ux8INryWRz8 (accessed on 11 March 2014).

[10] Dreze, J. and A. Sen (2013) An Uncertain Glory - India and Its Contradictions (London: AllenLane).
[11] Faure, E. et al (1972) Learning to Be: The World of Education Today and Tomorrow, Report ofthe Edgar Faure Commission (Paris: UNESCO).

[12] Hasan, A. (2012) 'Lifelong learning in Bangladeshin OECD and Developing Countries: An Interpretation andAssessment' in Aspin, D.N. (ed.) Second Springer International Handbook of Education(Portland, OR: Powell Books), vol. 26, chapter 29, pp. 471-497.

[13] Husén, T. (1986) The Learning Society Revisited (Oxford: Pergamon)

[14] Hutchins, R. M. (1970) The Learning Society (Harmondsworth: Penguin).

[15] Manzoor Ahmed, "Lifelong learning in Bangladeshin a Learning Society: Are Community Learning Centres the Vehicle?", International Development Policy | Revue internationale de politique de développement [Online], 5 | 2014, Online since 18 November 2014, connection on 04 March 2021. URL: http://journals.openedition.org/poldev/1782; DOI: https://doi.org/10.4000/poldev.1782

[16] Oyasu, K. and D. Riewpituk (2013) 'CLCs in the Asia Pacific Region', in Okayama ESD PromotionCommission, Education for Sustainable Development and Kminkan/Community Learning Centre(Okayama: Okayama University Press).

[17] Peterson, G. (1997) The Power of Words: Literacy and Revolution in South China, 1949-95(Vancouver: University of British Columbia Press).

[18] UIL (2009) The Belém Framework for Action, Sixth International Conference on Adult Education(CONFINTEA VI), Belem, Brazil, 1-4 December (Hamburg: UIL).

[19] UIL (2010) Shanghai International Forum on Lifelong Learning: Summary report of GeneralRapporteur, Shanghai, 19-21 May (Hamburg: UIL),http://uil.unesco.org/fileadmin/keydocuments/LifelongLearni ng/en/Summary\%20of\%20General\%20Rapporteur.pdf(accessed on 17 April 2014).

[20] UIL (UNESCO Institute for Lifelong Learning) (2013) Second Global Report on Adult Learningand Education - Rethinking Literacy (Hamburg: UIL).

[21] UNESCO (2011) 'Sustainability of Community Learning Centres: Community Ownership andSupport', Asia-Pacific Regional Action Research Studies (Bangkok: UNESCO Regional Office).

[22] UNESCO (2012) Community Learning Centres: Asia Pacific Regional Conference Report(Bangkok: UNESCO Regional Office). 